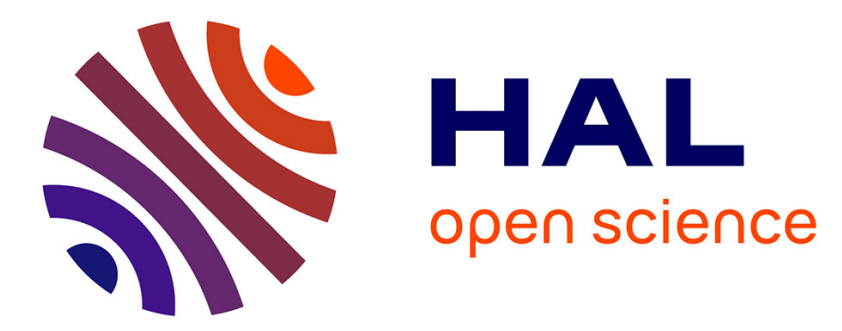

\title{
Modélisation des sols et des ouvrages avec le modèle Cam-Clay modifié
}

\author{
Philippe Mestat, Yvon Riou
}

\section{To cite this version:}

Philippe Mestat, Yvon Riou. Modélisation des sols et des ouvrages avec le modèle Cam-Clay modifié. Revue Française de Génie Civil , 2002, 6 (5), pp.801 - 815. 10.1080/12795119.2002.9692404 . hal01007070

\section{HAL Id: hal-01007070 \\ https://hal.science/hal-01007070}

Submitted on 3 Feb 2017

HAL is a multi-disciplinary open access archive for the deposit and dissemination of scientific research documents, whether they are published or not. The documents may come from teaching and research institutions in France or abroad, or from public or private research centers.
L'archive ouverte pluridisciplinaire HAL, est destinée au dépôt et à la diffusion de documents scientifiques de niveau recherche, publiés ou non, émanant des établissements d'enseignement et de recherche français ou étrangers, des laboratoires publics ou privés. 


\title{
Modélisation des sols et des ouvrages avec le modèle Cam-Clay modifié
}

\author{
Philippe Mestat* — Yvon Riou**
}

\author{
* Laboratoire Central des Ponts et Chaussées \\ 58, boulevard Lefebvre \\ F-75732 Paris cedex 15 \\ Philippe.Mestat@lcpc.fr \\ ** Ecole Centrale de Nantes, BP 92101 \\ F-44321 Nantes cedex 03 \\ Yvon.Riou@ec-nantes.fr
}

RÉSUMÉ. Une base de données sur la confrontation «calculs par éléments finis - mesures in situ » a été développée au Laboratoire Central des Ponts et Chaussées (LCPC). Cette base appelée MOMIS regroupe plus de 160 fiches concernant des ouvrages en terre et des ouvrages souterrains. Sa constitution repose sur une veille technologique dans le domaine de la modélisation par les éléments finis. Chaque fiche est le fruit de l'analyse d'une publication (article, communication ou rapport). Une exploitation particulière de cette base a permis de dresser un bilan quant à l'emploi du modèle de comportement "Cam-Clay modifié » pour les sols compressibles: analyse statistique des valeurs des paramètres et quantification des écarts entre les résultats numériques obtenus et les mesures réalisées sur les ouvrages réels.

ABSTRACT. A data base on confrontation of finite elements calculations and in situ measurements was developed with the Laboratoire Central des Ponts et Chaussées (LCPC). This base called MOMIS gathers more than 160 cards concerning of the ground works and the underground works. Its constitution rests on a technological survey in the field of modeling by finite elements. Each card is the result of the analysis of study (paper, communication or report). A specific exploitation of this data base made it possible to draw up an assessment about the use of "Cam-Clay modified" model for the compressible soils : statistical analysis values of the parameters and quantification of the gap between the numerical results and the measurements carried out on the real works.

Mots-ClÉs: modélisation, lois de comportement, détermination des paramètres, comparaison calculs-mesures, validation.

KEYWORDS: Modeling, constitutive relations, determination of parameters, calculationmeasurements comparison, validation. 


\section{Introduction}

La validation constitue une préoccupation commune aux concepteurs et aux utilisateurs de logiciels de calcul par éléments finis. Celle-ci doit prouver l'aptitude d'un logiciel et d'un modèle à résoudre de manière satisfaisante un problème d'ingénieur (ou de chercheur) dans des conditions proches de celles du projet. Différents aspects caractérisent la validation. On ne considère dans cet article que la « validation de modèle »; celle-ci est établie en constatant un écart raisonnable entre le comportement réel des ouvrages en vraie grandeur (mesures) et les résultats numériques fournis par le modèle (calculs). Malheureusement, cette confrontation est complexe, notamment dans le domaine de la géotechnique, car on en vient à tester simultanément un logiciel, un type d'analyse, un modèle théorique et la manière dont un utilisateur peut s'en servir (Mestat et Riou, 1999). Le modèle traduit alors la conception de la «réalité physique » qu'a l'ingénieur ou le chercheur à un moment donné et le compromis qu'il doit faire pour utiliser les outils numériques disponibles (notamment au niveau des hypothèses de calcul).

Curieusement malgré les nombreuses confrontations publiées dans le domaine de la géotechnique depuis près de trente ans, aucun véritable «bilan » n'a été dressé sur les écarts observés entre les modélisations par éléments finis et les mesures effectuées sur des ouvrages en vraie grandeur. Face à ce manque de repères nécessaires pour tirer des enseignements du passé et pour juger la capacité des lois de comportement et des logiciels à reproduire des situations complexes, la division de Mécanique des Sols, des Roches et de la Géologie de l'Ingénieur du LCPC a entrepris une veille technologique dans ce domaine (Mestat, 2000). Pour recueillir et traiter les informations liées aux expérimentations de référence, la base de données MOMIS (Modèles numériques d'Ouvrage et Mesures In Situ) a été développée (Mestat, 2001). Son exploitation permet entre autre de quantifier les performances d'une loi de comportement par rapport aux différents types de sol et d'ouvrages en interactions. Cette base de données comporte actuellement plus de 160 fiches concernant les remblais et les ouvrages souterrains. De nombreuses analyses statistiques sont possibles, notamment concernant les paramètres des lois de comportement. Quelques possibilités sont ainsi illustrées avec le modèle «Cam-Clay modifié », utilisé fréquemment pour décrire le comportement des sols compressibles.

\section{Conception de la base de données MOMIS}

L'exploitation des documents repérés lors de la veille technologique et relatant une confrontation "calculs par éléments finis - mesures sur ouvrages » consiste à réaliser une fiche synthétique et à renseigner diverses rubriques. Les informations sont ensuite stockées sous une forme informatique appropriée : il s'agit pour l'instant de fichiers créés avec le traitement de texte WORD97 et seules les modélisations de remblais sur sols compressibles et d'ouvrages souterrains ont été considérées. Chaque fiche est liée à une modélisation et rassemble des rubriques qui constituent 
autant d'étapes dans l'élaboration d'un modèle de calcul. Elles se rattachent à huit familles dans lesquelles sont reportées les informations suivantes :

- le type d'analyse (condition drainée, condition non drainée, consolidation, dynamique, cyclique) ;

- la nature des terrains rencontrés sur le site (et l'épaisseur des couches) ;

- la technique de construction, les dimensions réelles de l'ouvrage, une description de son environnement (nappe phréatique, ouvrages voisins, etc.) ;

- les lois de comportement pour les terrains naturels et les matériaux de construction (élasticité, élastoplasticité, élasto-visco-plasticité), la méthodologie de détermination des valeurs des paramètres ;

- le modèle de calcul (dimensions, type d'éléments finis, densité du maillage, conditions aux limites, sollicitations, pas de temps, phasage de construction, lois d'interface, etc.) et une figure représentant le maillage ;

- les courbes confrontant les résultats des calculs aux valeurs mesurées ;

- les conclusions sur la confrontation (écart maximum, erreurs relatives, etc.) ;

- les références bibliographiques des documents analysés.

Les données les plus anciennes remontent à 1972 et se répartissent plus ou moins régulièrement depuis cette date. Près de $70 \%$ des références ont paru dans les actes des congrès dédiés à la géotechnique ; $22 \%$ proviennent d'articles de revues et environ $8 \%$ de rapports ou de thèses. Pour un même ouvrage expérimental, on peut avoir plusieurs fiches, c'est notamment le cas lors de l'organisation d'un concours de prévisions à l'aveugle (prévisions de classe A au sens de Lambe (1973)). Celles-ci sont très rares et la grande majorité des calculs recensés sont des prévisions de classe B ou C pour lesquelles les auteurs disposent au préalable des valeurs mesurées, ce qui peut leur permettre d'adapter les paramètres de calcul aux observations.

L'objectif principal est de conserver la mémoire des modélisations par éléments finis, de tirer des enseignements sur la pratique en géotechnique, de fournir des ordres de grandeur des résultats des calculs et de quantifier l'erreur de modèle (différences entre valeurs calculées et mesurées).

\section{Type d'analyse et lois de comportement}

D'une manière générale, trois types d'analyse sont menés pour étudier les ouvrages de géotechnique : les analyses en condition non drainée, en condition drainée et les analyses en consolidation. Pour les remblais, la majorité des calculs prennent en compte les phénomènes de couplage hydromécanique de la consolidation (67\% des modèles de remblai introduits dans MOMIS). En revanche, pour les ouvrages souterrains, la majorité des modèles supposent des conditions drainées ( $75 \%$ des modèles de tunnels considérés dans MOMIS). Cette différence 
s'explique par la complexité de la modélisation du phasage des travaux pour les tunnels. On note toutefois une augmentation récente du nombre des modélisations en consolidation pour les ouvrages souterrains (15\% des références dans MOMIS).

Les modèles rhéologiques employés pour décrire le comportement des sols sont des lois élastiques linéaires ou non linéaires (12\%), élastoplastiques sans écrouissage (24\%), élastoplastiques avec écrouissage (46\%) et élasto-visco-plastiques (18\%). La tendance est à l'emploi de modèles plus évolués que la simple élastoplasticité parfaite. Les modèles élastoplastiques avec écrouissage sont notamment utilisés pour les sols compressibles. L'étude des références archivées dans MOMIS montre que le modèle «Cam-Clay modifié », développé à l'Université de Cambridge, est le plus utilisé pour les sols compressibles (35\% de toutes les références et $67 \%$ de toutes les lois élastoplastiques avec écrouissage). Les raisons de ce succès tiennent à la facilité avec laquelle ce modèle peut être introduit dans un code de calcul par éléments finis, au nombre réduit de ses paramètres et à leur détermination simple à partir des résultats d'essais classiques de mécanique des sols (œdomètre et essais triaxiaux).

\section{Présentation du modèle Cam-Clay modifié}

Roscoe et al. (1958) furent les premiers à établir, à l'Université de Cambridge (Royaume Uni), des relations générales de comportement des sols fondées sur la théorie de l'élastoplasticité avec écrouissage. Les modèles développés, connus sous le nom de "modèles Cam-Clay», sont destinés essentiellement à décrire le comportement des argiles reconstituées en laboratoire. Le modèle le plus utilisé est appelé «modèle Cam-Clay modifié », il est décrit brièvement ci-après.

Dans le plan (e, ln $\mathrm{p})$, e indice des vides et p pression moyenne, les résultats d'un essai de compression isotrope font apparaître des courbes qui peuvent être assimilées à des droites (figure 1) :

- une courbe de consolidation vierge, dite courbe $\lambda$, qui décrit le chargement au cours de l'essai ;

- une courbe de déchargement-rechargement, dite courbe $\kappa$, qui schématise un cycle de déchargement-rechargement.

Les courbes $\lambda$ et $\kappa$ sont définies par les équations suivantes :

$$
\text { courbe } \lambda: \quad \mathrm{e}=\mathrm{e}_{1}-\lambda \ln \frac{\mathrm{p}}{\mathrm{p}_{1}} \text { et courbe } \kappa: \quad \mathrm{e}=\mathrm{e}^{\mathrm{p}}-\kappa \ln \frac{\mathrm{p}}{\mathrm{p}_{1}} ;
$$

où $\mathrm{e}_{1}$ et $\mathrm{e}^{\mathrm{p}}$ correspondent aux valeurs obtenues pour une pression de référence $\mathrm{p}_{1}$ (prise égale à $1 \mathrm{kPa}) ; \mathrm{e}_{1}, \lambda$ et $\kappa$ sont trois paramètres du modèle. La figure 1 montre que la quantité $\mathrm{e}^{\mathrm{p}}$ évolue au cours du chargement (passage du point $\mathrm{A}_{0}$ au point $\mathrm{A}_{2}$ ) et qu'elle est liée à la limite du domaine d'élasticité actuel. Cette quantité peut donc être utilisée comme paramètre d'écrouissage. 


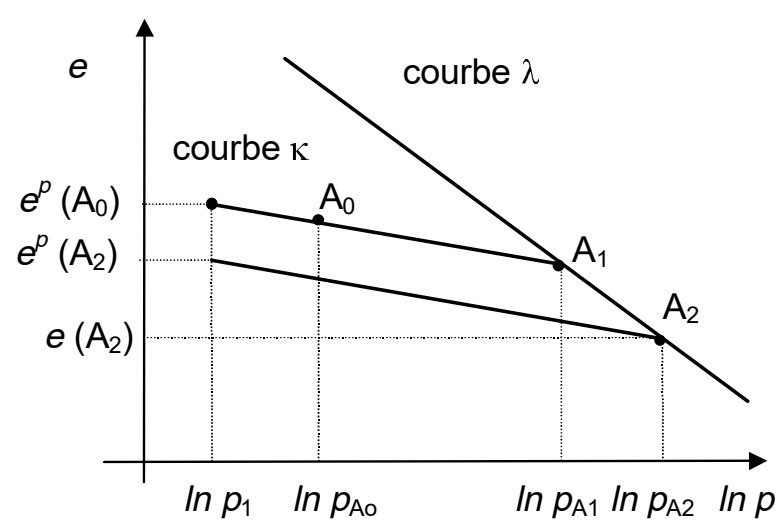

Figure 1. Représentation d'un essai de compression isotrope

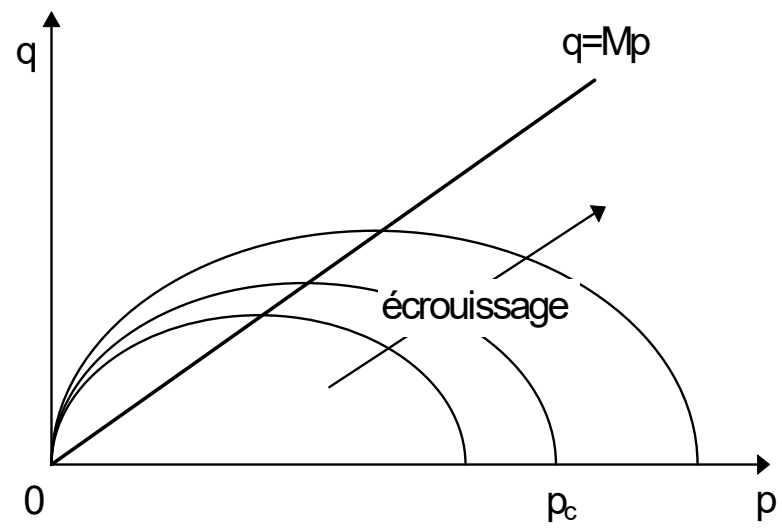

Figure 2. Surface de charge du modèle «Cam-Clay modifié» dans le plan $(p, q)$

Par ailleurs, l'analyse des résultats d'essais triaxiaux de cisaillement a montré que la rupture, " état critique », peut être caractérisée par les relations suivantes :

- un rapport de contraintes constant : $\mathrm{q}=\mathrm{Mp}$ ( $\mathrm{q}$, déviateur des contraintes) ;

- l'absence de variations de la déformation volumique : $\mathrm{d} \varepsilon_{\mathrm{v}}=0$;

- une courbe d'état critique dans le plan $(e, \ln p)$ de pente $\lambda: e=e_{c s}-\lambda \ln \frac{p}{p_{1}}$.

$M$ et $\mathrm{e}_{\mathrm{cs}}$ sont deux paramètres du modèle.

La surface de charge $\mathrm{F}$ est construite à partir d'une relation différentielle calée sur les résultats d'essais triaxiaux (notamment des essais de compression à pression moyenne constante et à rapport de contraintes $\mathrm{q} / \mathrm{p}$ fixé) et du principe de normalité (égalité de la fonction $\mathrm{F}$ et du potentiel plastique) :

$$
\mathrm{F}\left(\mathrm{p}, \mathrm{q}, \mathrm{p}_{\mathrm{c}}\right)=\frac{\mathrm{q}^{2}}{\mathrm{M}^{2} \mathrm{p}^{2}}+1-\frac{\mathrm{p}_{\mathrm{c}}}{\mathrm{p}}=0 .
$$

Le terme $\mathrm{p}_{\mathrm{c}}$ est la contrainte isotrope qui correspond au point d'intersection de la surface de charge avec l'axe de compression isotrope, limitant ainsi le domaine d'élasticité actuel (figure 2) ; cette pression peut être considérée comme la variable qui gouverne l'évolution de la surface de charge. La relation d'écrouissage lie l'indice $\mathrm{e}^{\mathrm{p}}$ (dont la variation est proportionnelle à la déformation volumique plastique) et la contrainte $p_{c}$; elle est obtenue en considérant les courbes $\lambda$ et $\kappa$ au point $\mathrm{p}_{\mathrm{c}}$. On a alors : $\frac{\mathrm{dp}_{\mathrm{c}}}{\mathrm{p}_{\mathrm{c}}}=\frac{1+\mathrm{e}_{\mathrm{o}}}{\lambda-\kappa} \mathrm{d} \varepsilon_{\mathrm{v}}^{\mathrm{p}}$ avec $\varepsilon_{\mathrm{v}}^{\mathrm{p}}=\varepsilon_{\mathrm{ij}}^{\mathrm{p}} \delta_{\mathrm{ij}}$.

L'élasticité associée au modèle «Cam-Clay modifié» est une élasticité non linéaire isotrope :

$$
\mathrm{d} \varepsilon_{\mathrm{ij}}^{\mathrm{e}}=\frac{1}{3 \mathrm{G}} \mathrm{d} \sigma_{\mathrm{ij}}+\frac{1}{3}\left(\frac{\kappa}{1+\mathrm{e}_{\mathrm{o}}} \frac{1}{\mathrm{p}}-\frac{1}{\mathrm{G}}\right) \mathrm{dp} \delta_{\mathrm{ij}}
$$


où $\mathrm{G}$ désigne le module de cisaillement et $\delta_{\mathrm{ij}}$, le tenseur de Kronecker.

Ce modèle comporte sept paramètres mécaniques $\left(M, \lambda, \kappa, e_{c s}, e_{1}, p_{1}, G\right)$, qui sont déduits de résultats d'essais triaxiaux (drainés et non drainés avec mesure de la pression interstitielle) et oedométriques classiques. L'état initial est caractérisé par les paramètres $\left(\mathrm{e}_{\mathrm{o}}, \mathrm{p}_{\mathrm{o}}, \mathrm{q}_{\mathrm{o}}\right)$.

Le chapitre suivant est consacré à l'estimation des performances du modèle «Cam-Clay modifié » dans la prévision du comportement des remblais sur sols compressibles et à l'analyse des variations des valeurs des paramètres.

\section{Ordres de grandeur des paramètres des lois de comportement}

Les modélisations répertoriées dans la base de données MOMIS décrivent les choix effectués par les géotechniciens responsables des calculs par éléments finis : élaboration du maillage, limites du domaine d'étude, type d'analyse, sollicitations, modèles de comportement, phasage des travaux, etc. Les résultats de ces choix ont été confrontés aux mesures sur les ouvrages. L'écart constaté permet de quantifier la performance d'un modèle.

Pour le modèle "Cam-Clay modifié » appliqué à la simulation des remblais sur sols compressibles, les figures 3 à 5 illustrent les écarts observés sur des modélisations de remblais (toutes prévisions confondues). Dans leur grande majorité, les valeurs calculées sont en accord raisonnable avec les mesures (tableaux 1 et 2). L'erreur de modèle est en moyenne proche de $25 \%$ pour les tassements, ce qui est tout à fait acceptable en géotechnique. En revanche, les erreurs sont généralement plus importantes pour les déplacements latéraux en pied de remblai qui sont souvent surévalués (figure 4) et pour les surpressions interstitielles (figure 5).

Si la confrontation calculs-mesures est jugée satisfaisante, cela signifie que les valeurs des paramètres employées permettent une bonne description des mouvements $\mathrm{du}$ sol et $\mathrm{du}$ fonctionnement de l'ouvrage. Ont été jugées satisfaisantes les modélisations dont l'erreur sur les tassements est inférieure à $25 \%$. Les valeurs des paramètres sont donc validées par la confrontation et constituent en quelque sorte le fruit d'une « analyse inverse », même s'il n'y a pas eu de véritable calage. L'intérêt consiste alors à déduire des informations sur les ordres de grandeur des paramètres : valeurs typiques et valeurs extrêmes. L'exploitation a concerné 123 séries de paramètres correspondant à des argiles et des limons (figure 6). À cause de leur très forte compressibilité, des grandes valeurs des paramètres $\left(\lambda, \kappa, \mathrm{e}_{\mathrm{cs}}, \mathrm{e}_{0}\right)$ et du peu de références utilisables, les tourbes ont été exclues des statistiques. 


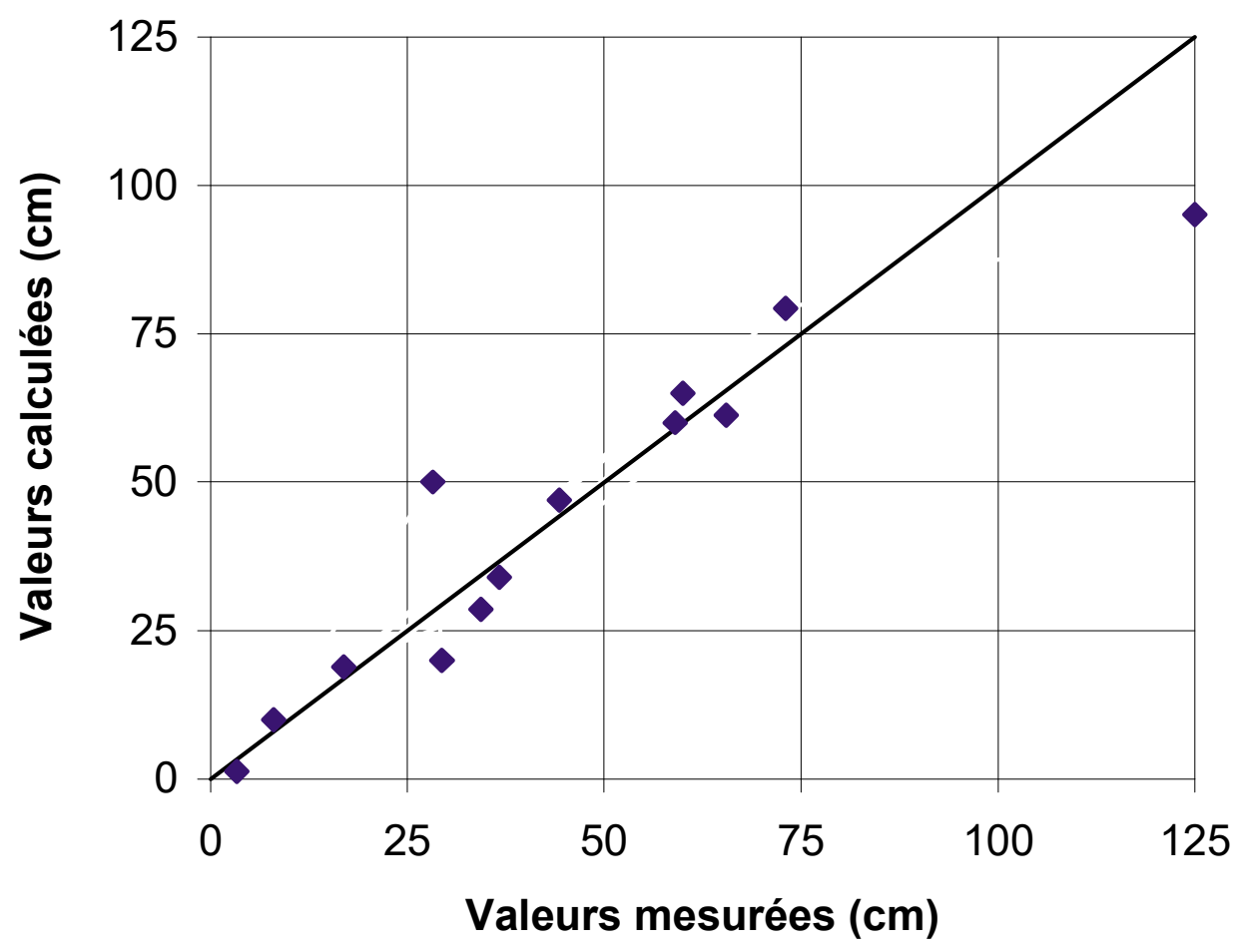

(a) Comparaison à la fin de la construction

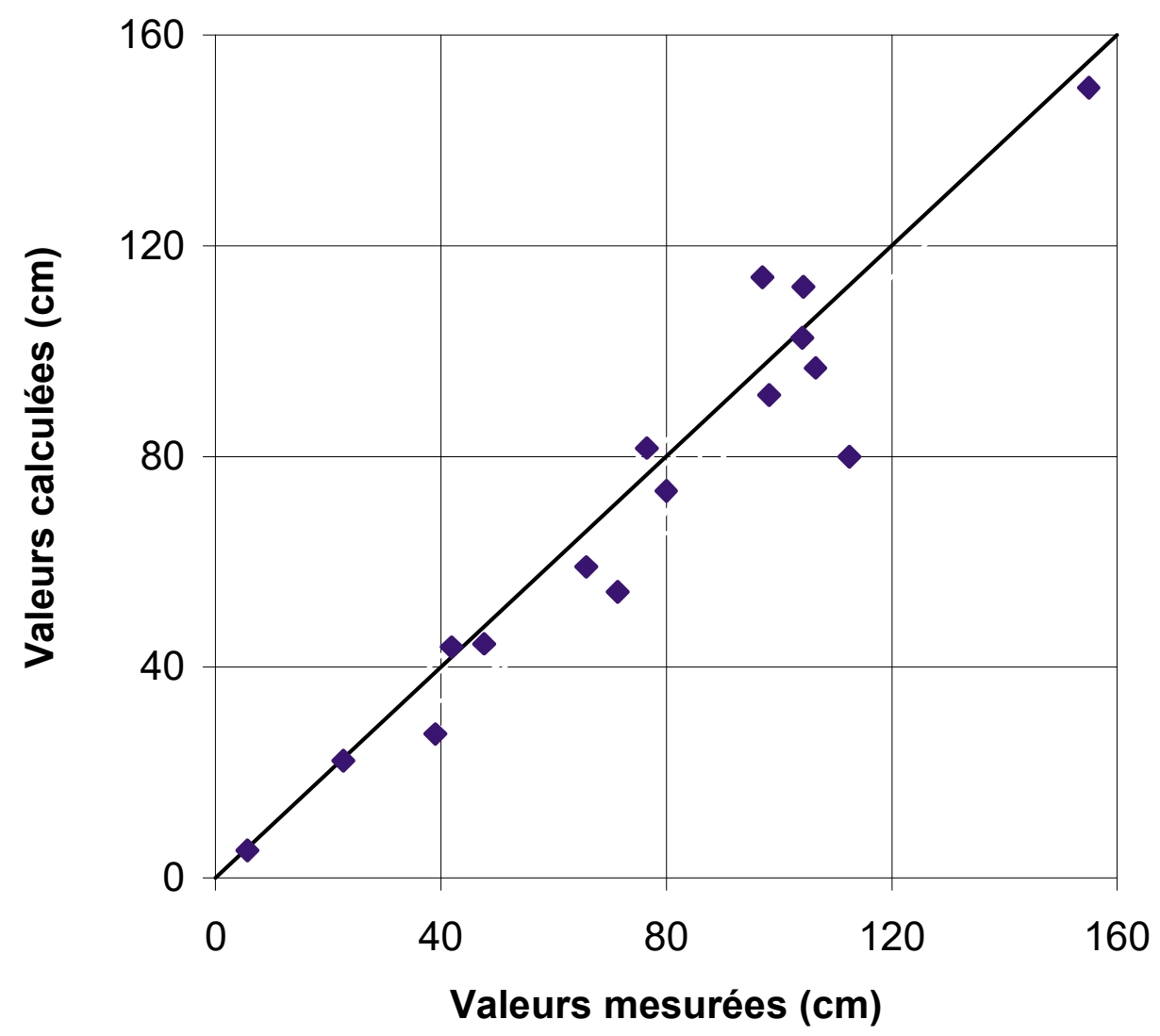

(b) Comparaison à long terme

Figure 3. Comparaison entre les tassements calculés et mesurés du sol de fondation dans l'axe du remblai (extraits de MOMIS) 


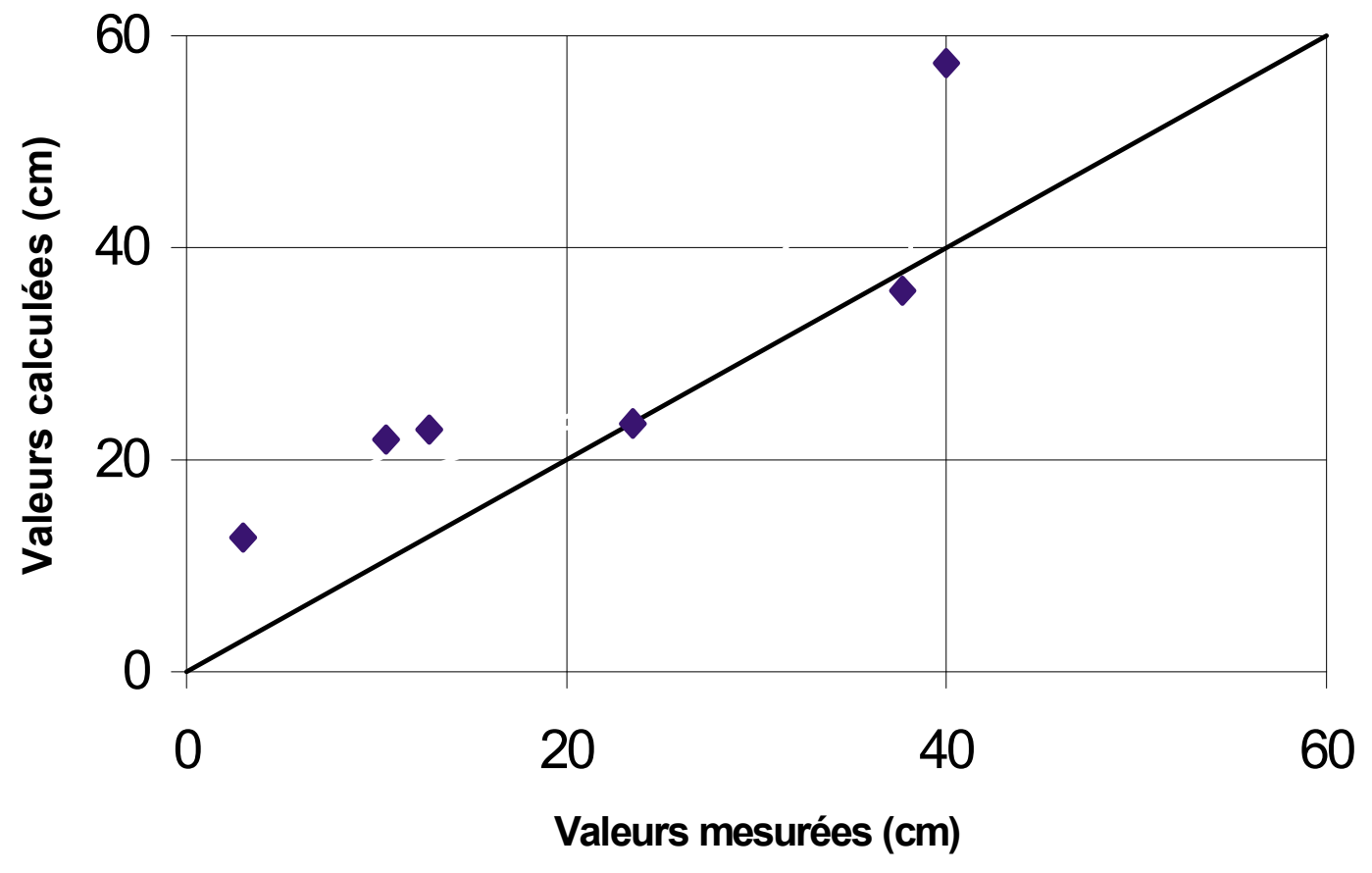

(a) Comparaison à la fin de la construction

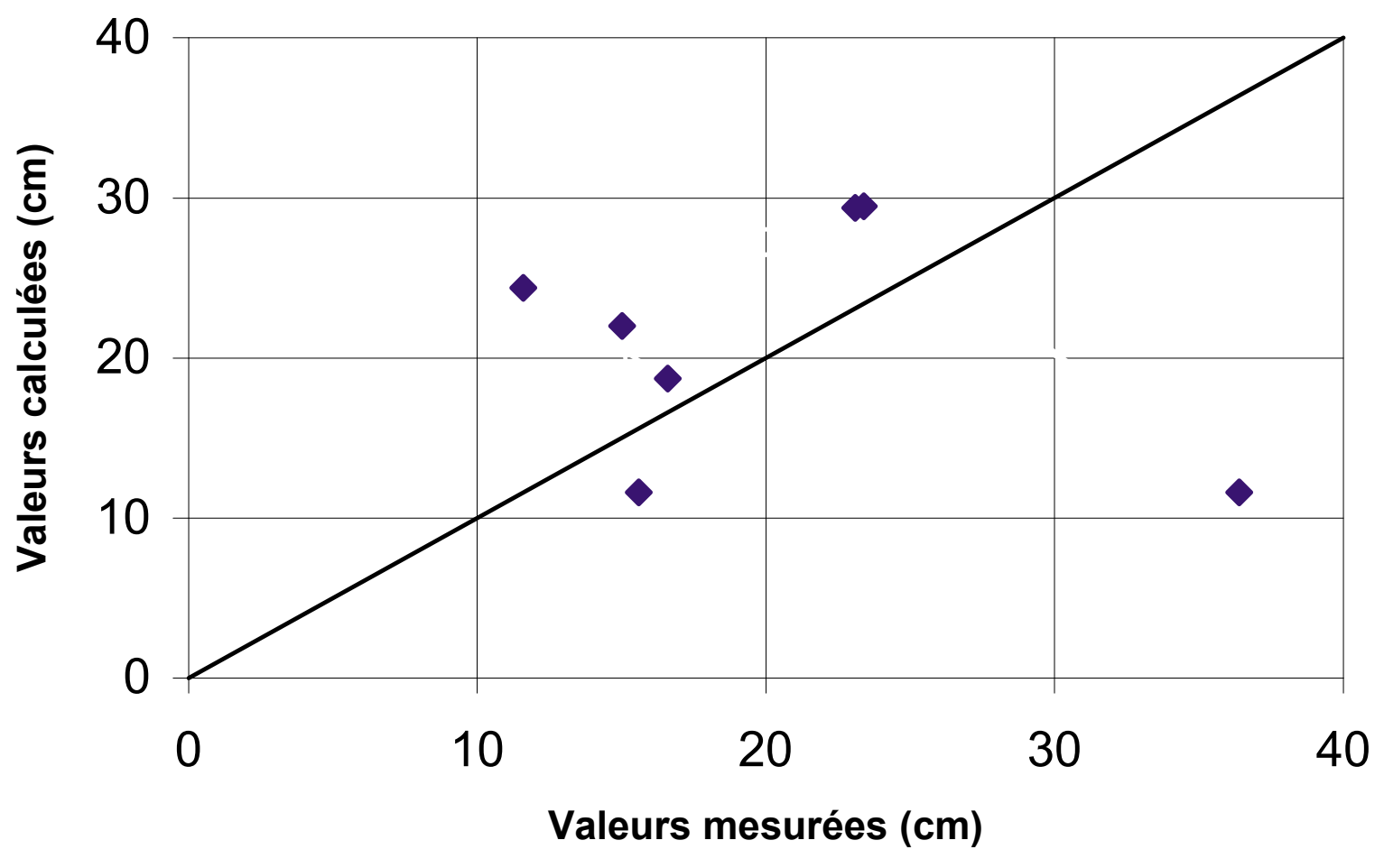

(b) Comparaison à long terme

Figure 4. Comparaison entre les déplacements latéraux maximum dans le sol en pied de remblai, calculés et mesurés (extraits de MOMIS) 


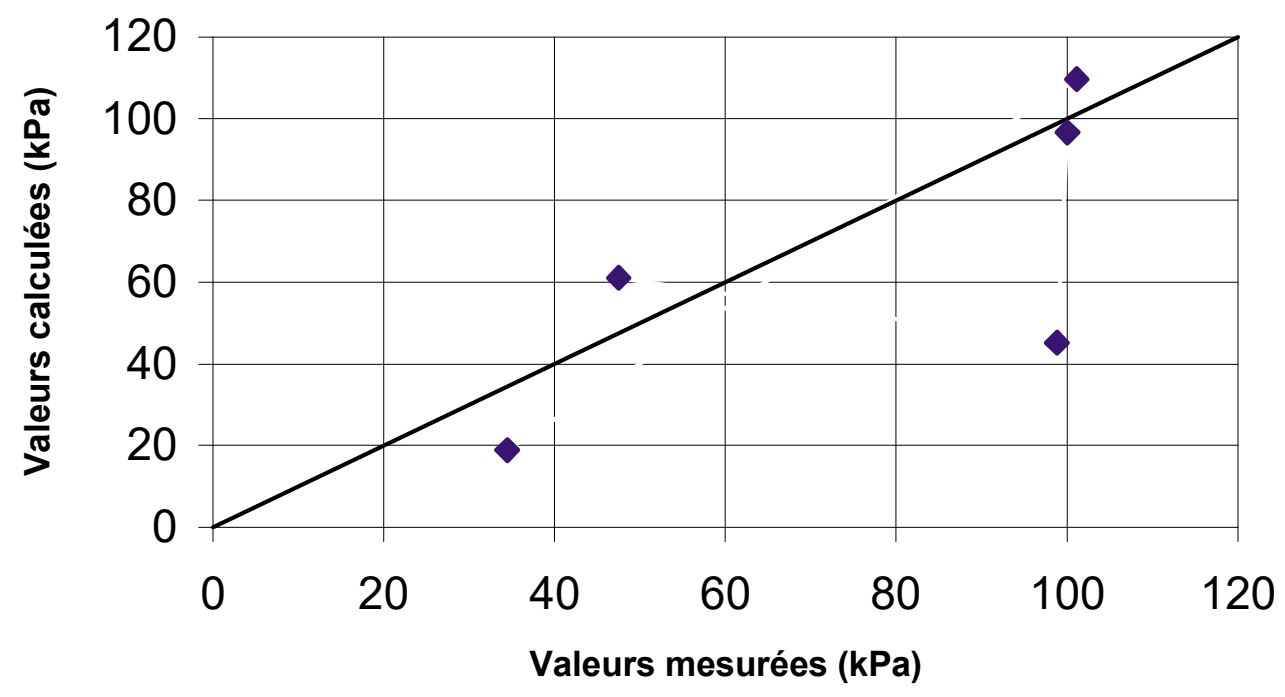

Figure 5. Comparaison des surpressions interstitielles calculées et mesurées à la fin de la construction (extraits de MOMIS)

Une analyse en fréquence montre que certains paramètres $(\kappa, \lambda, M$ et $\nu)$ ont des plages de variation relativement étroites avec des pics marqués. Les variations concernant les paramètres $\kappa$ et $\lambda$ sont similaires ; cette remarque est confirmée par la figure 7 qui démontre une certaine « corrélation » entre les valeurs des paramètres $\kappa$ et $\lambda$. On devine une tendance autour de la relation $: \kappa=\lambda / 10$.

En revanche, les autres paramètres présentent des fréquences plus étalées (figure 6). Les ordres de grandeur ainsi mis en évidence ne constituent qu'une indication, les sols concernés par les confrontations calculs-mesures n'étant pas représentatifs de tous les sols naturels. C'est le mérite de la base MOMIS que de fournir également la description des sols en relation avec les valeurs des paramètres du modèle. L'ingénieur ou le chercheur peut alors approfondir sa réflexion en prenant connaissance d'une ou de plusieurs références proches du projet qu'il a à traiter. L'existence de ces références ne doit en aucun cas l'empêcher de réaliser et d'interpréter tous les essais nécessaires à la mise en données de son problème.

\section{Conclusions}

La division de Mécanique des Sols, des Roches et de la Géologie de l'Ingénieur du LCPC mène depuis plusieurs années une action de "veille technologique » dans le domaine de la confrontation des résultats de modèles d'éléments finis avec des mesures réalisées sur des ouvrages réels. Les informations dégagées de l'analyse bibliographique ont été regroupées dans une base de données appelée MOMIS. Celleci contient plus de 160 références concernant les remblais et tunnels. Une exploitation particulière de MOMIS a permis de quantifier les performances du modèle « Cam-Clay modifié » pour les sols compressibles dont le caractère anisotrope n'est pas fortement prononcé et de caractériser les ordres de grandeur des valeurs des paramètres. 
Cette étude statistique sur les valeurs de paramètres «validées » avait plusieurs objectifs :

- fournir des intervalles de variations « réalistes » aux développeurs de logiciel pour établir des contrôles sur les données et attirer l'attention d'un utilisateur sur des valeurs aberrantes ;

- aider l'ingénieur ou le chercheur dans sa recherche des «bonnes» valeurs de paramètres ;

- constituer un point de départ pour un processus d'optimisation des valeurs de paramètres.

La base de données MOMIS devrait également permettre dans un proche avenir de comparer les valeurs des paramètres obtenues en laboratoire et in situ avec les valeurs retenues pour la modélisation numérique (c'est notamment le cas des modules de déformabilité et des coefficients de perméabilité pour lesquels la prise en compte d'essais in situ est essentielle).

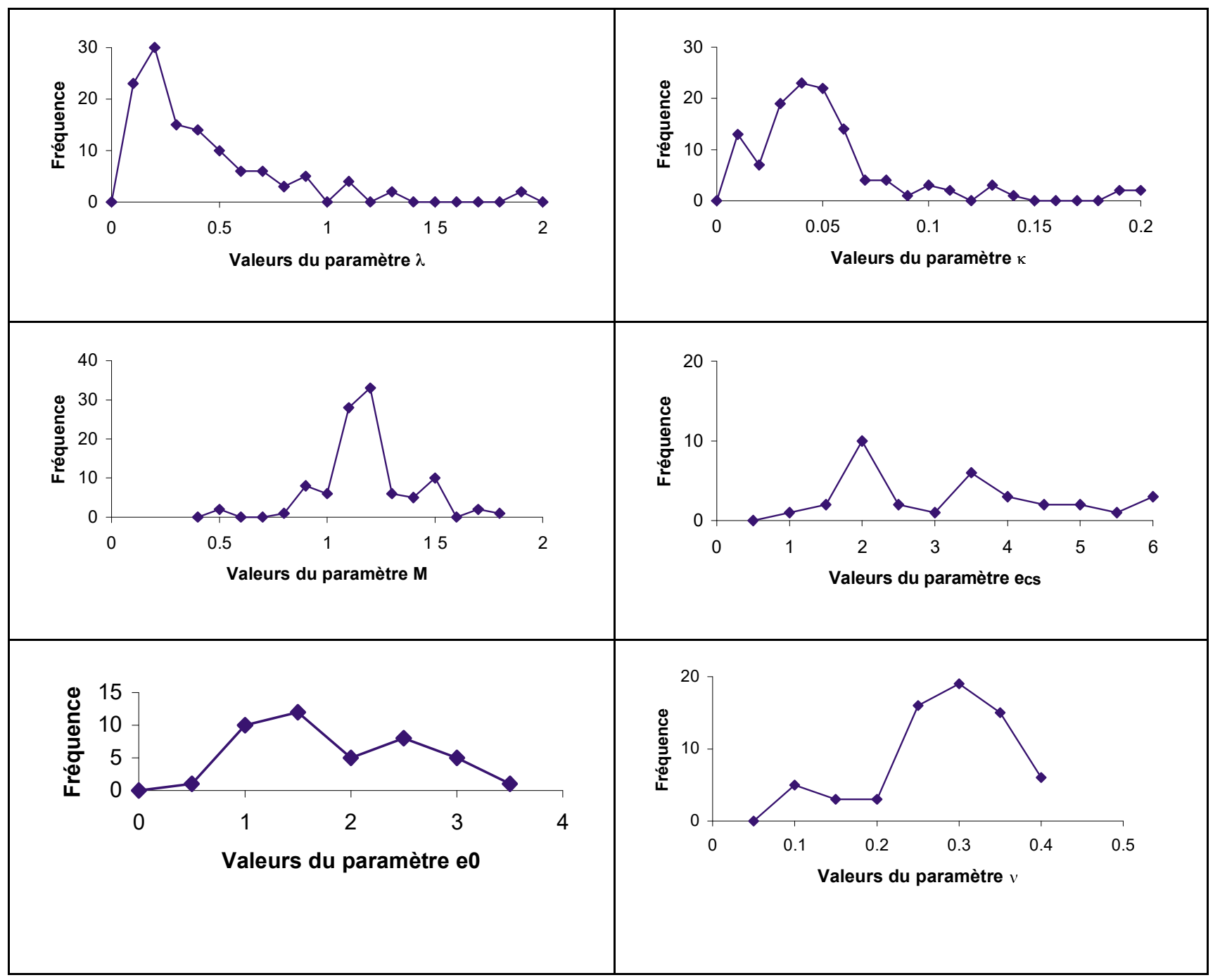

Figure 6. Distribution statistique des valeurs des paramètres du modèle "CamClay modifié », pour les sols compressibles à l'exception des tourbes (extraits de MOMIS) 


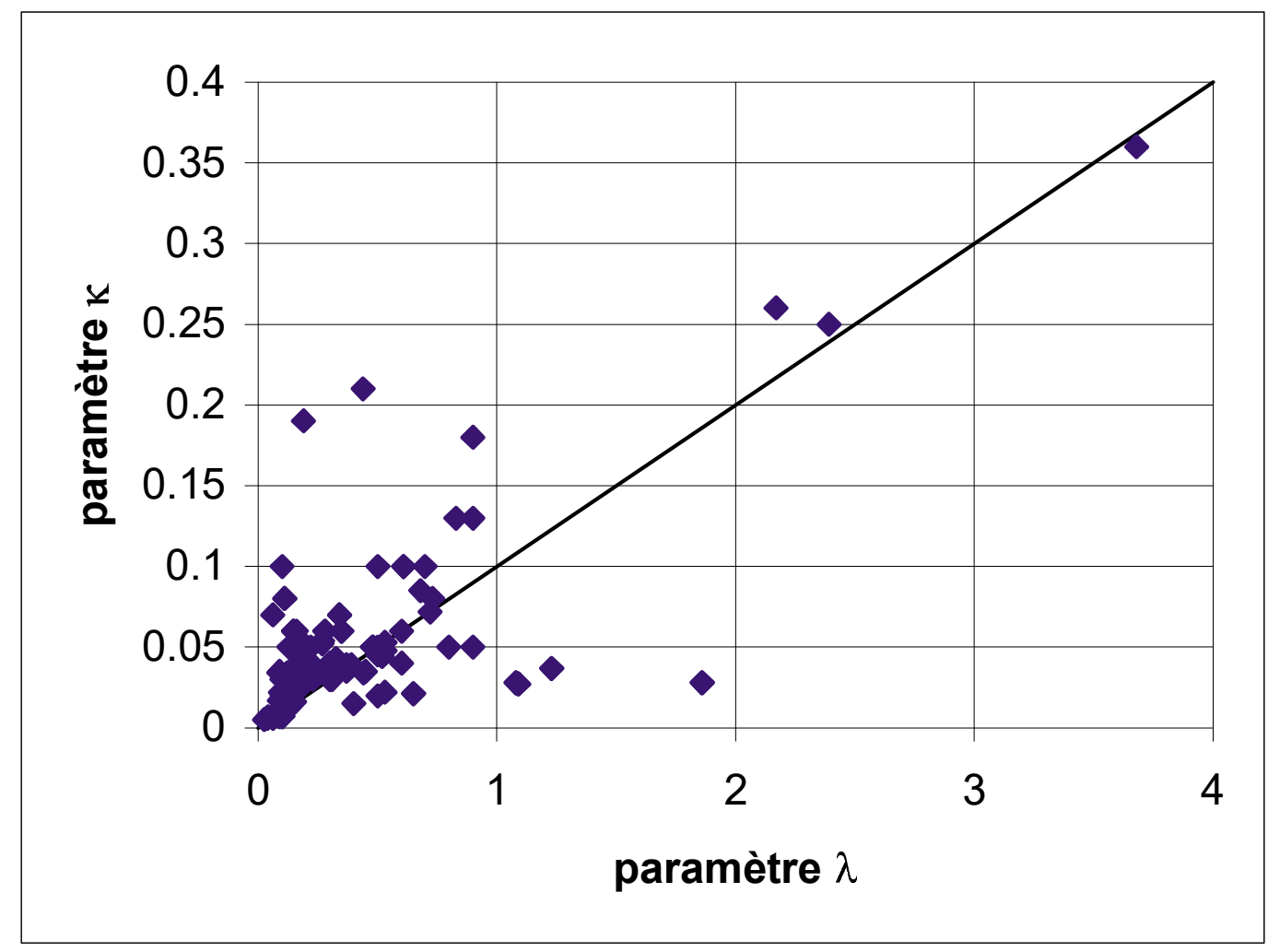

Figure 7. Corrélation entre les paramètres $\lambda$ et $\kappa$ du modèle Cam-Clay modifié (extraits de MOMIS)

\section{Bibliographie}

Aalto A., Rekonen R., Lojander M. (1998), «The calculations on Haarajoki test embankment with the finite element program PLAXIS $6.31 »$, Application of Numerical Methods to Geotechnical Problems - NUMGE98, Cividini (ed), Springer, p. 37-46.

Ali F.H., Mohamad R. (1989), « Critical state parametric study on control embankment », Int. Symp. on Trial Embankments on Malaysian Marine Clays, Kuala Lumpur, p. 30.1-30.5.

Ali F.H., Hashim R. (1991), "A road embankment on soft clay : field behaviour and prediction ", Computer Methods and Advances in Geomechanics, Beer et al. (eds), Balkema, p. 943-948.

Almeida M.S.S., Ramalho-Ortigao (1982), «Performance and finite element analyses of a trial embankment on soft clay », Int Symp. on Numerical Models in Geomechanics, Zurich, 13-17 septembre 1982, Balkema, p. 548-558.

Balasubramaniam A.S., Phien-Wej N., Indraratna B., Bergado D.T. (1989), «Predicted behaviour of a test embankment on Malaysian marine clay», Int. Symp. on Trial Embankments on Malaysian Marine Clays, Kuala Lumpur, p. 1.1-1.8.

Borja R.I., Hsieh H.S., Kavazanjian E. (1990), «Double-yield-surface model. II: implementation and verification », Journal of Geotechnical Engineering, ASCE, vol. 116, $\mathrm{n}^{\circ}$ 9, p. 1402-1421. 
Brugger P.J., de Souza Soares de Almeida M., Sandroni S.S., Lacerda W.A. (1998), "Numerical analysis of the breakwater construction of Sergipe Harbour, Brazil», Canadian Geotechnical Journal, vol. 35, p. 1018-1031.

Chai J.C., Sakajo S., Miura N. (1994), Stability analysis of embankment on soft ground (a case study), Soils and Foundations, vol. 34, $\mathrm{n}^{\circ} 2$, p. 107-114.

Colleselli F., Cortellazzo G. (1994a), «Boundary conditions in the behaviour of large embankments », Numerical Methods in Geotechnical Engineering, Smith (ed), Balkema, p. $333-339$.

Colleselli F., Cortellazzo G. (1994b), «Foundation behaviour of a levee in the Po Delta », Prediction versus Performance in Geotechnical Engineering, Balasubramaniam et al. (eds), Balkema, p. 15-20.

Hayashi H., Nishikawa J., Yamaguchi S., Mitachi T., Fukuda F. (1998), «Finite element analysis of peaty ground using a Cam Clay model », Problematic Soils, Yanagisawa et al. (eds), Balkema, p. 123-127.

Indraratna B., Balasubramaniam A.S., Balachandran S. (1992), «Performance of test embankment constructed to failure on soft marine clay », Journal of Geotechnical Engineering, ASCE, vol. 118, ${ }^{\circ} 1$, p. 12-33.

Kavazanjian E., Borja R.I., Jong H.L. (1985), « Time-dependant deformations in clay soils », 11th International Conference on Soil Mechanics and Foundation Engineering, San Francisco, vol. 2, p. 535-538.

Koehorst B.A.N., The B.H.P., Teunissen J.A.M., Lojander M., Näätänen A. (1992), « The trial embankments in Vaasa, Finland. A simulation with the Adachi-Oka creep model », Numerical Models in Geomechanics, NUMOG IV, Pande et Pietruszczak (eds), Balkema, p. 767-776.

Ladd C.C., Whittle A.J., Legaspi D.E. (1994), «Stress-deformation behaviour of an embankment on Boston blue clay », Vertical and Horizontal Deformations of Foundations and Embankments, Geotechnical Special publications, ${ }^{\circ} 40$, Yeung et Félio (eds), ASCE, p. 1730-1759.

Lambe T. W. (1973), « Prediction in soil engineering », Géotechnique, vol. 23, n 2, p. 149-202.

Magnan J.P., Humbert P., Belkeziz A., Mouratidis A. (1982), « Finite element analysis of soil consolidation, with special reference to the case of strain hardening elastoplastic stressstrain models "), 4th Int. Conf. on Numerical Methods in Geomechanics, Eisenstein (ed), Balkema, p. 327-336.

Mestat Ph., Riou Y. (1999), «A propos des benchmarks en géotechnique », Revue Française de Génie Civil, vol. 3, n 7-8, p. 657-672.

Mestat Ph. (2000), De la rhéologie des sols à la modélisation des ouvrages géotechniques. Etudes et Recherches des Laboratoires des Ponts et Chaussées, série géotechnique, GT 67, 236 pages.

Mestat Ph. (2001), « An overview on 25 years of numerical modeling of test embankments and tunnels », Computer Methods and Advances in Geomechanics, Desai et al. (eds), Balkema, p. 1521-1526. 
Mitachi T., Fukuda F. (1998), «Applicability of Cam Clay model as a constitutive relation for consolidation-deformation coupled three dimensional FE analysis for highly organic soft ground », Problematic Soils, Yanagisawa et al. (eds), Balkema, p. 65-68.

Roscoe K.H., Schofield A.N., Wroth C.P. (1958), «On the yielding of soils », Géotechnique, vol. $8, n^{\circ} 1$, p. 22-53.

Vepsäläinen P., Arkima O., Lojander M., Nätänen A. (1991), «The trial embankments in Vaasa and Paimio, Finlande », Xth European Conference on Soil Mechanics and Foundation Engineering, Balkema, p. 633-640.

Wroth C.P., Simpson B. (1972), «An induced failure at a trial embankment. Part II Finite element computations », Performance of Earth and Earth-supported Structures, Purdue University, ASCE, vol. 1, Part 1, p. 65-79. 


\begin{tabular}{|c|c|c|c|c|c|c|c|c|c|c|}
\hline 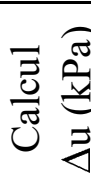 & & $\frac{1}{6}$ & & $\hat{\circ}$ & 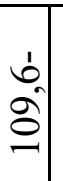 & & & s. & & \\
\hline 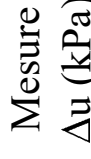 & & 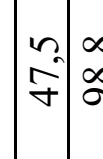 & & & $\overrightarrow{0}$ & & & : & ' & \\
\hline 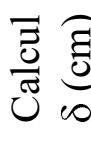 & & \begin{tabular}{lll}
0 \\
\hdashline
\end{tabular} & & $\begin{array}{l}+ \\
i \\
i n\end{array}$ & & & & & $\mid \frac{2}{2}$ & \\
\hline 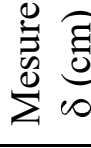 & & $m$ & & f & & & & & 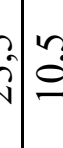 & \\
\hline & $\therefore=$ & 0 & & 8 & $\frac{2}{6}$ & & & $\infty_{\infty}$ & $=$ & \\
\hline$\frac{b^{2}}{20}$ & \begin{tabular}{c}
$a$ \\
\hdashline \\
$c$
\end{tabular} & & & in & $\mid \begin{array}{l}n \\
\vdots \\
0\end{array}$ & & 8 & 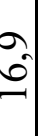 & $\approx$ & \\
\hline & & $=$ & & & 部 & & & 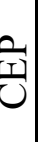 & 䆑 & \\
\hline 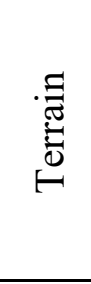 & 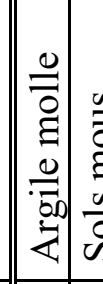 & 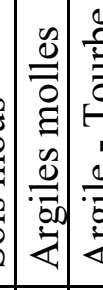 & 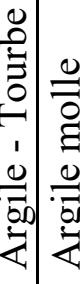 & 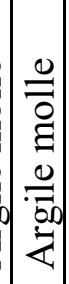 & 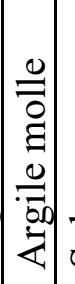 & & 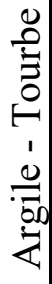 & 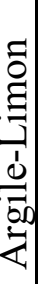 & 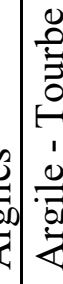 & \\
\hline 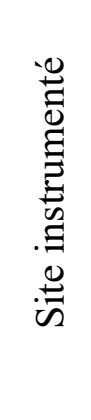 & 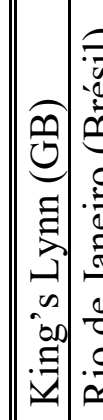 & 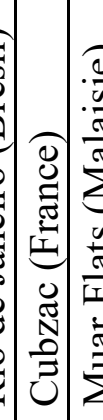 & 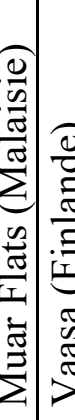 & & $\mid$ & & 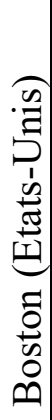 & 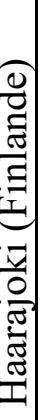 & 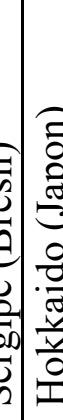 & \\
\hline 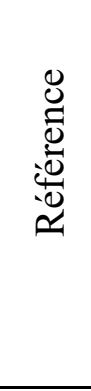 & 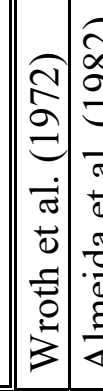 & 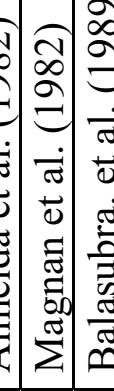 & 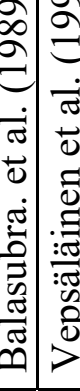 & 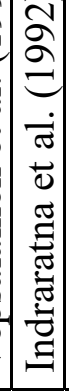 & 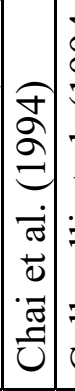 & & 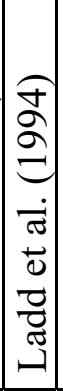 & בְ⿱ & 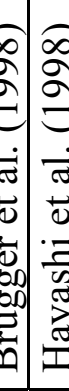 & \\
\hline
\end{tabular}




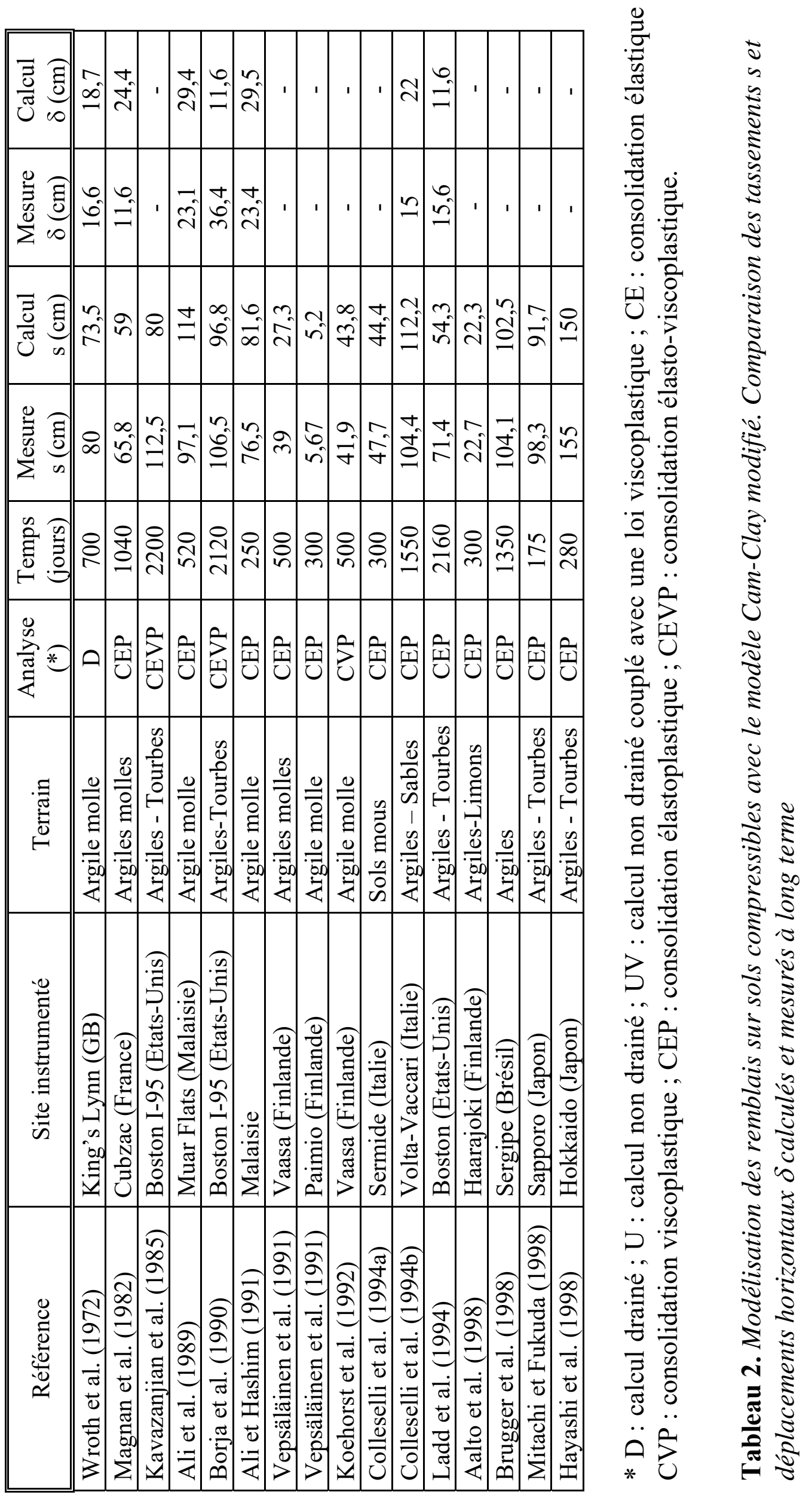

